\title{
Observation of kinks and antikinks in colloidal monolayers driven across ordered surfaces
}

\author{
Thomas Bohlein ${ }^{1,2}$, Jules Mikhael ${ }^{1}$ and Clemens Bechinger ${ }^{1,2 \star}$
}

\begin{abstract}
Friction between solids is responsible for many phenomena such as earthquakes, wear or crack propagation ${ }^{1-4}$. Unlike macroscopic objects, which only touch locally owing to their surface roughness, spatially extended contacts form between atomically flat surfaces. They are described by the FrenkelKontorova model, which considers a monolayer of interacting particles on a periodic substrate potential ${ }^{5-8}$. In addition to the well-known stick-slip motion, such models also predict the formation of kinks and antikinks ${ }^{9-12}$, which greatly reduce the friction between the monolayer and the substrate. Here, we report the direct observation of kinks and antikinks in a two-dimensional colloidal crystal that is driven across different types of ordered substrate. We show that the frictional properties only depend on the number and density of such excitations, which propagate through the monolayer along the direction of the applied force. In addition, we also observe kinks on quasicrystalline surfaces, which demonstrates that they are not limited to periodic substrates but occur under more general conditions.
\end{abstract}

Friction is important in our daily life and it is not surprising that systematic investigations date back more than 300 years. According to Amontons and Coulomb, friction between solids is proportional to the normal force but independent of the contact area. This intriguing result was explained by realizing that macroscopic objects touch at asperities that are deformed by the normal force ${ }^{13}$. A different situation occurs when atomically flat surfaces slide against each other, as for example encountered in micro- or nanoelectromechanical systems. Then, extended contacts arise and the degree of commensurability between the surfaces determines the friction. For commensurate conditions, a dissipative stick-slip motion is typically observed ${ }^{14}$. In contrast, at incommensurate interfaces, atomic friction studies revealed a superlubrication regime, where the friction coefficient vanishes ${ }^{15,16}$. This behaviour can be explained by simple mechanical models such as the FrenkelKontorova model, a generalized Prandtl-Tomlinson scheme or the double-chain model ${ }^{17-20}$. In the Frenkel-Kontorova approach the interface between two solids is described by a monolayer of elastically interacting beads on a periodic substrate potential ${ }^{5-8}$. In addition to stick-slip motion, the Frenkel-Kontorova model also predicts the formation of topological solitons, so-called kinks and antikinks ${ }^{9-12}$. These excitations are believed to dominate the frictional properties at atomic length scales because they provide an efficient mechanism for mass transport; so far, such excitations have never been observed in sliding friction experiments ${ }^{21}$.

Here, we report the observation of kinks and antikinks in a colloidal system that is driven across commensurate and incommensurate substrate potentials. We use highly charged polystyrene spheres with radius $R=1.95 \mu \mathrm{m}$, which are suspended in water. In the presence of gravitational and optical forces, they form a two-dimensional triangular crystal at the bottom of the sample cell, whose particle trajectories can be determined with video microscopy (Methods). Substrate potentials are created with interfering laser beams, which lead to ordered periodic energy landscapes acting on the colloids (Fig. 1a; ref. 22). To apply lateral forces to the colloidal crystal, the sample cell is translated with velocity $u$, which leads to a Stokes force $F \sim u$ acting on each particle (Methods).

The friction of a colloidal monolayer can be characterized by its mobility $\mu=v / F$ where $v$ is the mean colloidal velocity at driving force $F$. Figure $1 \mathrm{~b}$ shows the results for a triangular colloidal crystal with lattice spacing $a=5.7 \mu \mathrm{m}$ driven across triangular substrates along one of the substrate symmetry directions. The lattice constant $s$ varied between $s=5.7$ and $4.8 \mu \mathrm{m}$ and the laser intensity was kept constant at $2.65 \mu \mathrm{W} \mu \mathrm{m}^{-2}$ to allow for a direct comparison. For commensurate conditions ( $a=s$, green symbols), the crystal remains pinned for $F<49 \mathrm{fN}$, where $F$ is below the static friction force (Fig. 1c). A further increase of $F$ leads first to a nonlinear behaviour of the crystal's velocity, which corresponds to its partial depinning from the substrate (Fig. 1d). Finally, complete depinning from the substrate occurs and the crystal slides freely across the surface (Fig. 1e). In this regime, the crystal's velocity becomes identical to that on a flat surface (dashed line). At incommensurate conditions, $s=5.2 \mu \mathrm{m}$ (red symbols), a similar behaviour is found but static friction is greatly reduced. For even stronger incommensurate conditions, that is $s=4.8 \mu \mathrm{m}$ (blue symbols), no static friction is observed within our resolution, which corresponds to superlubric sliding. This strong dependence of superlubric behaviour on the substrate lattice spacing is in agreement with atomic friction force experiments on incommensurate surfaces ${ }^{15,23}$. We also carried out friction experiments on nonperiodic substrate potentials with quasiperiodic order, which are strongly incommensurate with the periodic monolayer ${ }^{22}$. Here, we find almost the same behaviour as for triangular substrates with $s=4.8 \mu \mathrm{m}$, which is consistent with the small friction coefficient on atomic quasicrystalline surfaces reported from experiments and simulations ${ }^{24-26}$. It should be mentioned that within our experimental resolution no hysteresis effects in the data of Fig. 1b were observed on increasing or decreasing $F$.

Figure $2 \mathrm{a}-\mathrm{c}$ shows snapshots of the particles' drift velocities under commensurate conditions (Methods). When $F$ exceeds the static friction force, fast (dark blue) and slow (light blue) particles assemble in clusters (Fig. 2b). Further increase of $F$ leads to the extension of such clusters in the $y$ direction (Fig. 2c). We also calculated the local crystal deformation which is obtained from the Voronoi cell areas (Fig. 2d-f; Methods). Obviously, regions of lattice compressions (dark green) and expansions (light green) agree with areas of fast and slow particles, respectively. Remarkably, such spatial variations in the particle properties are also observed

\footnotetext{
12. Physikalisches Institut, Universität Stuttgart, Pfaffenwaldring 57, 70569 Stuttgart, Germany, ${ }^{2}$ Max-Planck-Institut für Intelligente Systeme, Heisenbergstraße 3, 70569 Stuttgart, Germany. ${ }^{\star}$ e-mail: c.bechinger@physik.uni-stuttgart.de.
} 

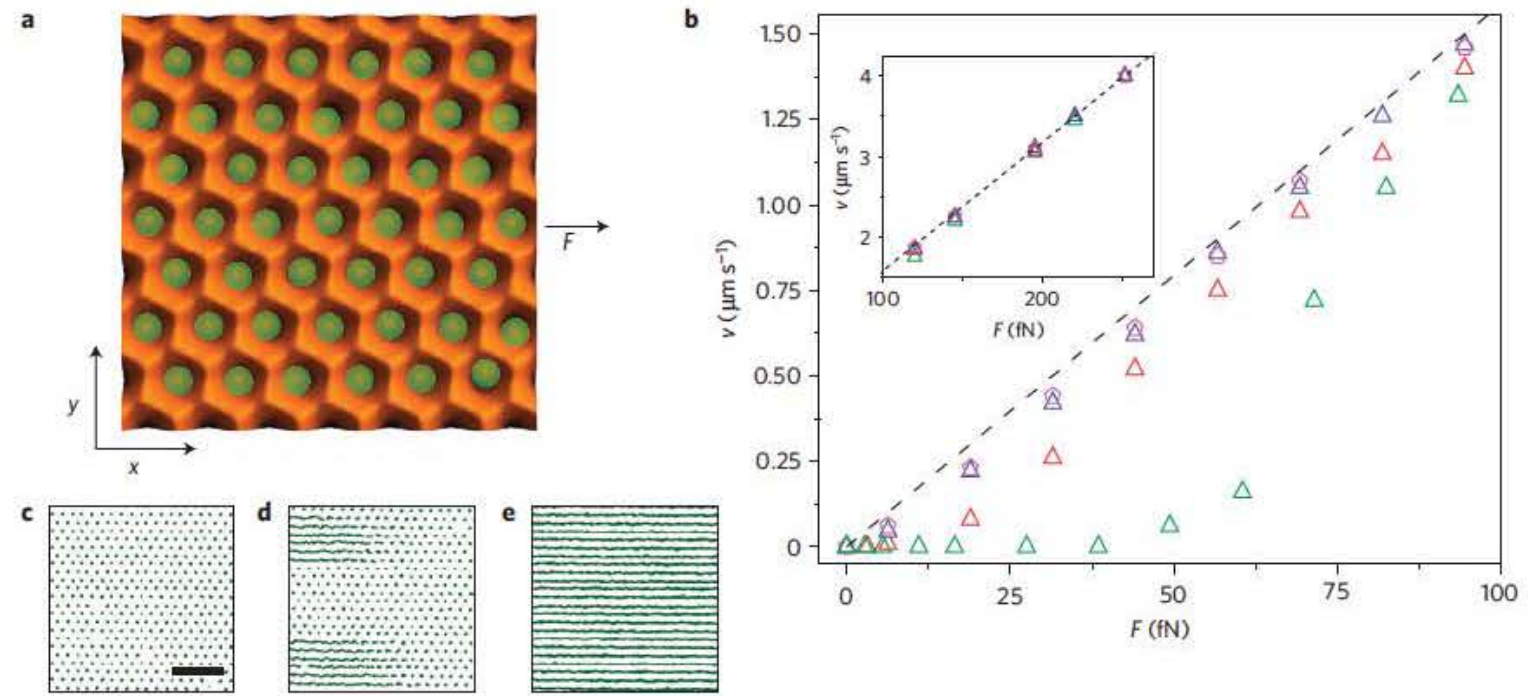

Figure 1 | Colloidal monolayers driven on optical interference patterns. a, Sketch of a colloidal monolayer driven with force $F$ across an energy landscape created by interference of laser beams. The potential strength and length scales of these potentials can be adjusted by the laser intensity and the angle under which the laser beams intersect. $\mathbf{b}$, Mean colloidal velocity $v$ of a crystalline monolayer with lattice constant $a=5.7 \mu \mathrm{m}$ versus $F$ for commensurate ( $a=s=5.7 \mu \mathrm{m}$, green), incommensurate $(s=5.2 \mu \mathrm{m}$, red; $s=4.8 \mu \mathrm{m}$, blue) and quasiperiodic (magenta) substrates. The dashed line corresponds to free sliding on a flat substrate. Inset: $v$ for larger $F$ where $v \sim F$. c-e, Trajectories for commensurate conditions and $F=0 \mathrm{fN}($ c), $49 \mathrm{fN}($ d) and $82 \mathrm{fN}($ (e). Scale bar, $30 \mu \mathrm{m}$.
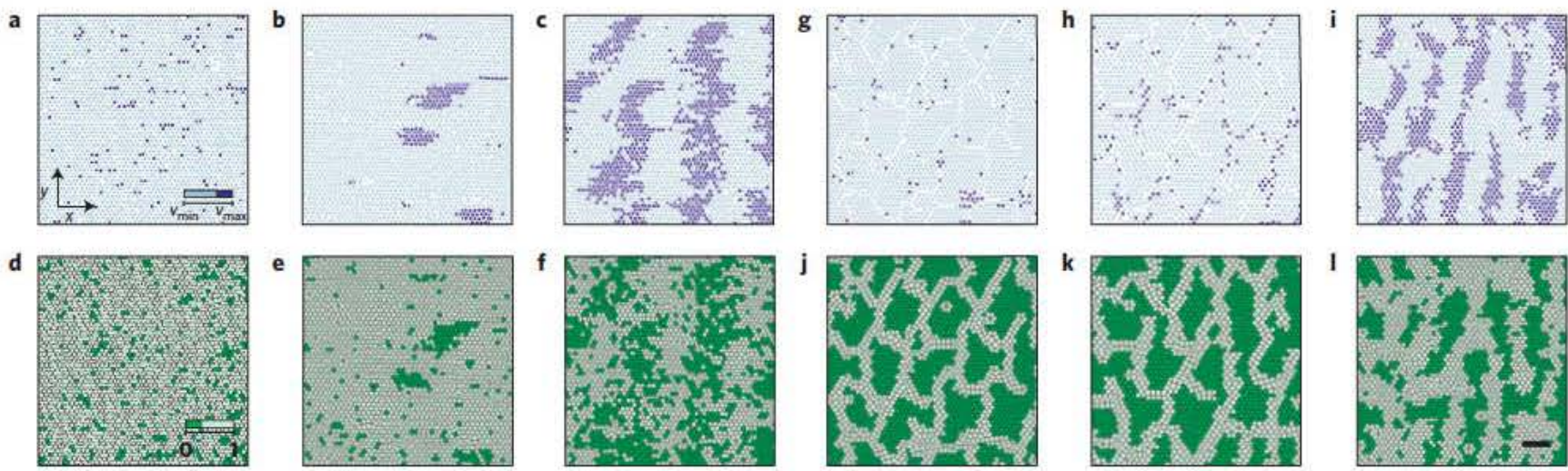

Figure 2 | Particle velocity and lattice deformation. a-c, Snapshots of particle velocities for commensurate conditions $(a=s=5.7 \mu \mathrm{m})$ and $F=0 \mathrm{fN}(\mathbf{a})$, $49 \mathrm{fN}(\mathrm{b})$ and $82 \mathrm{fN}$ (c). Fast and slow particles are marked in dark and light blue depending on whether their velocities are above or below $70 \%$ of the maximum particle velocity. $\mathbf{d}-\mathbf{f}$, Voronoi tessellation with colour-coded areas of the Voronoi cells: light (large) to dark green (small). $\mathbf{g}-\mathbf{I}$, Corresponding plots for incommensurate conditions $a>s=5.2 \mu \mathrm{m}$ and driving forces $0 \mathrm{fN}(\mathbf{g}, \mathbf{j}), 19 \mathrm{fN}(\mathbf{h}, \mathbf{k})$ and $82 \mathrm{fN}(\mathbf{i}, \mathbf{l})$. The laser intensity is identical to that in Fig. $1 \mathrm{~b}$. Scale bar, $30 \mu \mathrm{m}$.

when the crystal is depinned from the substrate (Fig. $2 \mathrm{c}, \mathrm{f}$ ). Only at much higher forces $F \cong 200 \mathrm{fN}$ (data not shown) does the colloidal monolayer slide undistorted across the substrate. Corresponding plots for incommensurate conditions $(s=5.2 \mu \mathrm{m})$ show a network of colloidal lattice expansions (white areas in Fig. $2 \mathrm{~g}-1$ ), which also occur in atomic systems and result from the release of accumulated stress ${ }^{27}$. At these domain walls, the next-neighbour distances are slightly larger and lead to higher particle mobilities (Fig. $2 \mathrm{~h}, \mathrm{k}$ ). Similar to the above, with increasing $F$, the domain walls become deformed into a stripe-like pattern; however, here the fast particles (dark blue) are at local lattice expansions (light green). For $s=4.8 \mu \mathrm{m}$ a similar behaviour with a finer network of domain walls is observed ${ }^{28}$.

Figure 3a shows the temporal behaviour of a compression zone while travelling across a commensurate surface for $F=40 \mathrm{fN}$ (see also Supplementary Movies S1 and S2). It propagates at about $3.4 \mu \mathrm{m} \mathrm{s}^{-1}$ in the direction of $F$ (to the right) without major changes in its shape. The $x$ component of particle trajectories (marked in orange in Fig. 3a) shows that, during passage of the compression zone, each colloid is displaced by one substrate lattice constant with a small time delay along the particle chain. Such a correlated particle motion is characteristic for a running kink ${ }^{29,30}$, which is illustrated for a one-dimensional system in Fig. 3c. In two-dimensional systems, such kinks are predicted to extend also perpendicular to $F$ with a small tilt with respect to the $y$ direction ${ }^{30}$. This is in excellent agreement with our observations (Fig. 3a); with increasing $F$ the tilt becomes smaller and the kinks aligned almost perpendicular to $F$ (Fig. 2c,f; see also Supplementary Movie S3; ref. 30).

Owing to the finite range of the pair interaction, the kinks observed in our experiments also expand in the $x$ direction. From Fig. 3a this width $L$ is estimated to be about $L=8-9$ in units of the substrate lattice constant $s$. Because each particle is displaced by one lattice spacing $s$ (dashed horizontal lines) when passed by a kink, the time a particle needs to surmount a potential barrier is 

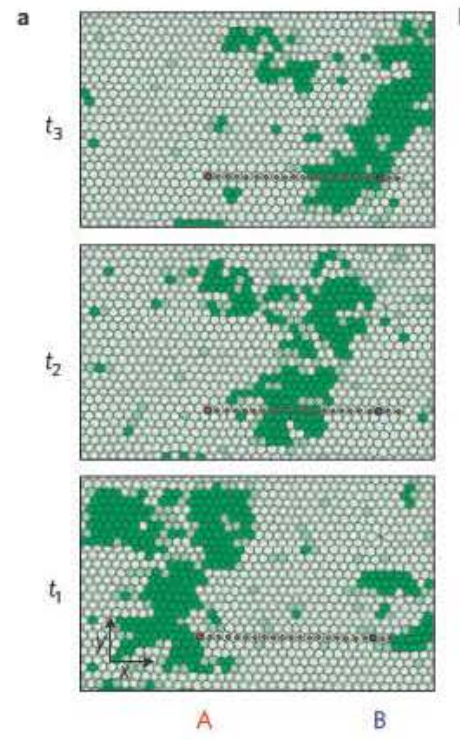

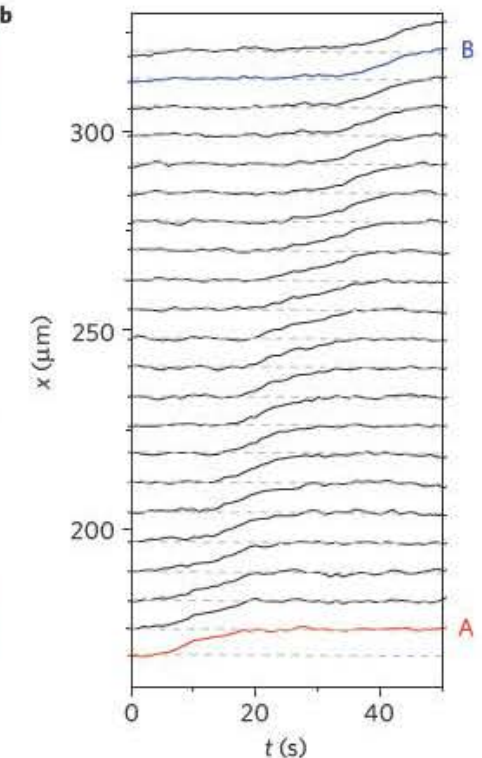

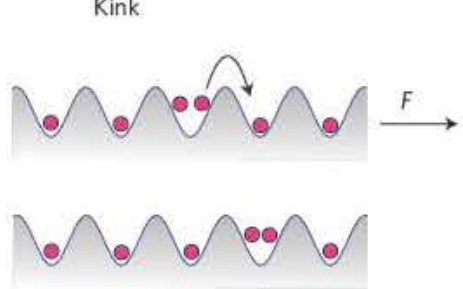

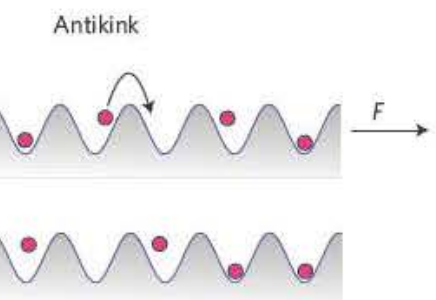

Figure 3 | Kink propagation through a colloidal monolayer, a, Propagating compression zone (dark green) on a commensurate substrate for $F=40 \mathrm{fN}$. The time interval between the snapshots is $13 \mathrm{~s}$. $\mathbf{b}, x$ component of particle trajectories marked in a (orange dots). Each particle is displaced by one lattice site (dashed lines) to the right during passage of the compression zone. $\mathbf{c}$, Sketch of a kink in a particle chain on a one-dimensional substrate potential. A kink forms when two particles in the same potential well lead to a local compression in the particle chain. The mobility of kinks is larger than that of a single particle because the energy barrier required for kink motion is smaller than the substrate amplitude. Therefore, mass transport through kinks provides an effective mechanism for the motion of the chain in the direction of F, d, Sketch of an antikink, which corresponds to a local expansion in the particle chain. Antikinks move in the direction opposite to that of $F$.

Table 1 | Comparison of calculated and measured velocities of colloidal monolayers.

Commensurate $(a=s=5.7 \mu \mathrm{m})$

\begin{tabular}{|c|c|c|c|c|}
\hline$F(\mathrm{fN})$ & $n_{\text {kink }}$ & $v_{\text {kink }}\left(\mu \mathrm{m} \mathrm{s}^{-1}\right)$ & $v_{\text {kink }} n_{\text {kink }} / N\left(\mu \mathrm{m} \mathrm{s}^{-1}\right)$ & $v\left(\mu \mathrm{ms}^{-1}\right)$ \\
\hline 120 & $4.2 \pm 0.4$ & $29.2 \pm 1.4$ & $1.75 \pm 0.19$ & 1.76 \\
\hline 145 & $4.2 \pm 0.4$ & $37.5 \pm 2.3$ & $2.24 \pm 0.26$ & 2.2 \\
\hline 195 & $4.2 \pm 0.4$ & $48.0 \pm 3.8$ & $2.87 \pm 0.36$ & 3.1 \\
\hline \multicolumn{5}{|c|}{ Incommensurate $(a>s=5.2 \mu \mathrm{m})$} \\
\hline$F(\mathrm{fN})$ & $n_{\text {antikink }}$ & $v_{\text {antikink }}\left(\mu \mathrm{m} \mathrm{s}^{-1}\right)$ & $v_{\text {antikink }} n_{\text {antikink }} / \mathrm{N}^{-1}\left(\mu \mathrm{ms}^{-1}\right)$ & $v\left(\mu \mathrm{ms}^{-1}\right)$ \\
\hline 120 & $8.5 \pm 1.2$ & $15.8 \pm 0.8$ & $1.92 \pm 0.28$ & 1.84 \\
\hline 145 & $8.5 \pm 1.2$ & $17.6 \pm 1.0$ & $2.14 \pm 0.33$ & 2.25 \\
\hline 195 & $8.5 \pm 1.2$ & $28.3 \pm 2.1$ & $3.43 \pm 0.55$ & 3.1 \\
\hline
\end{tabular}

Mean velocity of a colloidal monolayer according to equation (1) calculated from the number and velocity of kinks and antikinks for commensurate and incommensurate conditions. The values compare well with the corresponding experimentally determined values.

$t_{\mathrm{h}} \approx L s / v_{\text {kink }}$ where $v_{\text {kink }}$ is the kink velocity. This leads to $v_{\text {kink }} \approx L v_{h}$ with $v_{h}=s / t_{h}$. From Fig. 3b we determined $v_{h} \approx 0.42 \mu \mathrm{m} \mathrm{s}^{-1}$, which yields $v_{\text {kink }}=3.36-3.78 \mu \mathrm{m} \mathrm{s}^{-1}$, in good agreement with Fig. $3 \mathrm{a}$. In commensurate conditions, the creation of kinks sets in immediately when $F$ exceeds the static friction force. When $F$ is further increased, the number of kinks first increases and eventually saturates, after the monolayer has completely depinned from the substrate. Assuming that particles are mobile only inside kinks and pinned otherwise, the mean particle velocity of a driven one-dimensional particle chain is

$$
v=v_{\text {kink }} n_{\text {kink }} / N
$$

where $n_{\text {kink }}$ and $N$ correspond to the number of kinks and the total number of particles, respectively. We have tested this relation for commensurate conditions in the range $F=120,145$ and $195 \mathrm{fN}$ (inset in Fig. 1b). At these values, the kinks extend in the $y$ direction over the entire field of view and equation (1) should apply. Indeed, the mean velocity of the monolayer calculated from equation (1) is within our experimental errors in good agreement with the measurement of $v$ taken from Fig. $1 \mathrm{~b}$.

The Frenkel-Kontorova model also predicts the occurrence of antikinks, which correspond to local expansions of the particle lattice (Fig. 3d). In contrast to kinks, antikinks move in the direction opposite to that of $F$. Although in commensurate, onedimensional Frenkel-Kontorova models kinks and antikinks form pairwise, only a few antikinks were found in our experiments under commensurate conditions. We attribute this to a small lattice distortion of the colloidal monolayer in presence of a driving force. Antikinks are easily observed under incommensurate conditions for $a>s$ (Fig. 2g-l; see also Supplementary Movie S4). Here, they coincide with the local lattice expansions at domain walls, which already form for $F=0$. As no energy for antikink formation 

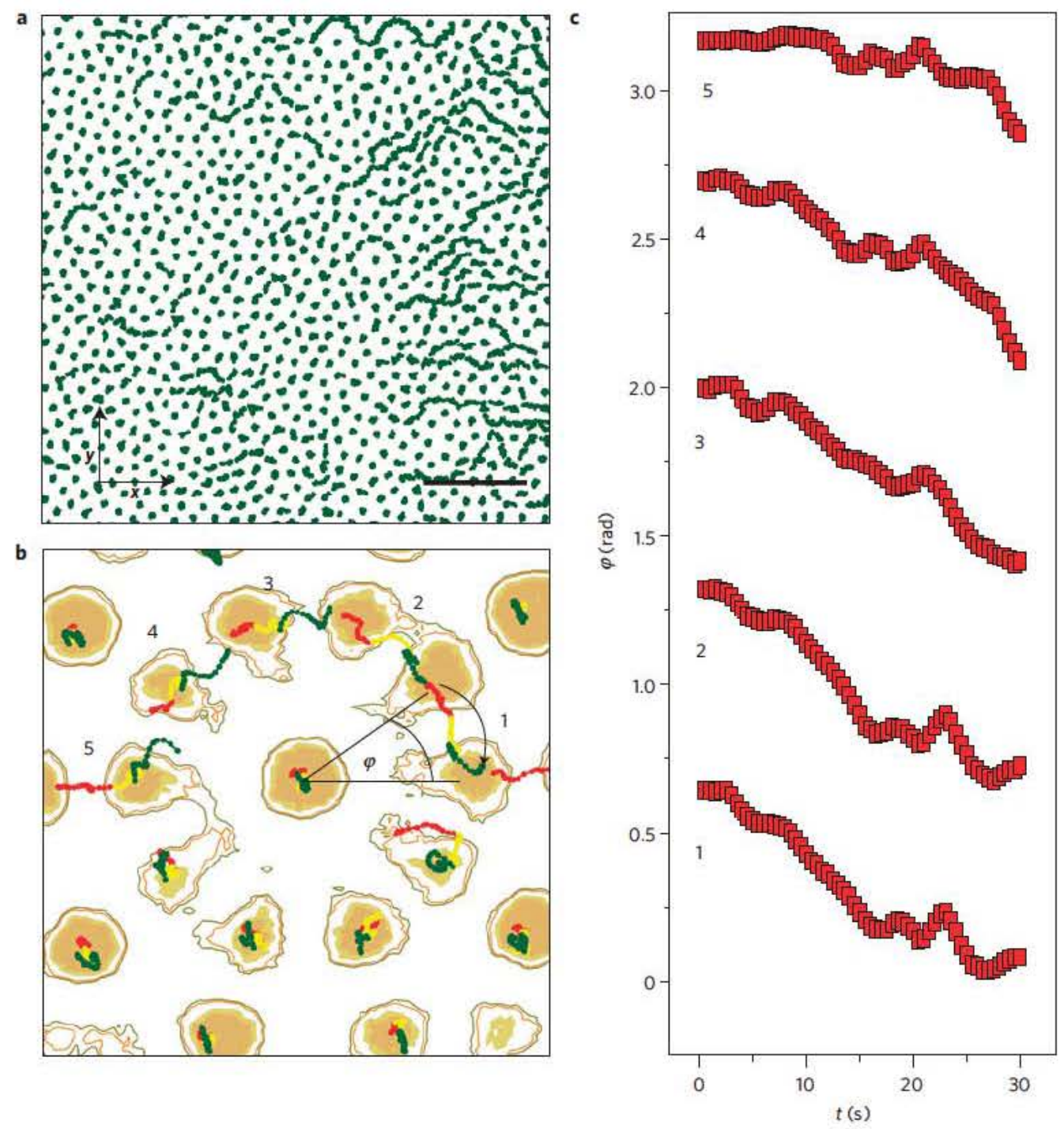

Figure 4 | Localized kinks on quasiperiodic substrates. a, Particle trajectories on a quasiperiodic substrate for $F=46 \mathrm{fN}$ (applied to the right), which is close to the depinning transition. Scale bar, $30 \mu \mathrm{m}$. b. Magnification of particle trajectories close to a highly symmetric motive (flower) of the substrate potential. The trajectories are colour coded and change from red to yellow to green as time proceeds. $c$, Angular coordinates of the particle trajectories shown in b, which suggest a correlated kink-like particle motion.

is required in this case, this easily explains the greatly reduced static friction force when compared with commensurate conditions. Because equation (1) is expected to hold also for antikinks, in Table 1 we have also calculated $v$ from the corresponding number of antikinks for incommensurate conditions $(s=5.2 \mu \mathrm{m})$. Within our experimental errors good agreement with the direct measurements of the mean colloidal velocity is obtained.

In contrast to periodic substrate potentials, little is known about the formation of kinks and antikinks on non-periodic substrate potentials ${ }^{23,31}$.Therefore, we carried out further experiments on quasiperiodic surface potentials at higher laser intensities $\left(6.5 \mu \mathrm{W} \mu \mathrm{m}^{-2}\right)$ to shift the depinning transition to higher values of $F$ (Fig. 1b). At these values, the colloids partly adopted decagonal order, leading to tenfold peaks in the structure factor (data not shown). Fig. 4a shows the trajectories for $F=46 \mathrm{fN}$, where a fraction of colloids becomes mobile (partial depinning). When compared with periodic potentials, however, two important differences occur: first the particles do not move in straight lines but take curved paths, and second chains of mobile particles do not propagate across the entire monolayer but remain rather localized. A comparison of the position of mobile particles with the underlying substrate potential shows a coincidence with specific motives in the substrate (Fig. 4b). Such motives are known from atomic quasicrystals as flowers and exhibit tenfold rotational symmetry. The angular component of the trajectories of the particles labelled in Fig. $4 \mathrm{~b}$ shows a strongly correlated motion while propagating across a flower (Fig. 4c). Because the substrate potential is locally periodic along such curved trajectories, this allows for a kink-like mechanism even in the presence of non-periodic surfaces. This may also explain the observed low friction coefficient on quasicrystalline surfaces. In addition, such particle motion at the edge of flowers also avoids particles becoming trapped in the deep potential minima at the flower centres ${ }^{31}$.

In addition to the observation of kinks and antikinks, our experimental set-up should also allow for the observation of the Aubry transition or transition of breaking analyticity. This occurs under incommensurate conditions for irrational fractions $a / s$ and when the ratio between the pair potential and the particle substrate potential exceeds a critical value $e^{32}$. According to numerical simulations, then the monolayer can change its configuration without energy cost by non-rigid particle displacements, which should lead to frictionless sliding ${ }^{21,23}$. 


\section{Methods}

We used carboxylated polystyrene particles with $R=1.95 \mu \mathrm{m}$ and a polydispersity of $2.7 \%$. Owing to functionalization with $-\mathrm{COOH}$ groups the particles are negatively charged in water and interact through a screened Coulomb repulsion. The Debye screening length was determined to be $160 \mathrm{~nm}$ (ref. 33). Optical interference patterns were created using an $18 \mathrm{~W}$ solid-state laser whose beam was divided into three or five beams and overlapped in the sample plane. The length scales of the patterns were adjusted by the angle of incidence. A further laser beam $(514 \mathrm{~nm})$ is vertically incident on the sample cell and pushes the colloids towards the bottom of the sample cell. This reduces out-of-plane particle fluctuations to less than $5 \%$ of the particle diameter.

To apply lateral forces to the colloidal monolayer the sample cell was mounted on a piezo table which can be laterally translated with nanometre accuracy. When the sample cell moves with velocity $\mathbf{u}$, this leads to viscous Stokes forces acting on each particle $\mathbf{F}=\eta_{\text {eff }} \mathbf{u}$. The effective drag coefficient $\eta_{\text {eff }}$ is determined by measuring the short-time particle diffusion coefficient $D$ within the monolayer and exploiting the Einstein relation $\eta_{\text {eff }}=k_{\mathrm{B}} T / D$

From the particle trajectories we construct the Voronoi tessellation and colour code the Voronoi cells according to their area from dark (small) to light green (large). Particle drift velocities were obtained from smoothed particle trajectories, where a moving average routine was applied to minimize the effects of Brownian motion. We have chosen a width of the averaging window of $2 \mathrm{~s}$ but similar results are obtained for slightly different window sizes. The relation between the applied force $F$ and the mean colloidal velocity $v$ is given by the mobility of the colloidal monolayer $\mu=v / F=v / \eta_{\text {eff }} u$.

Owing to kink propagation through the monolayer, the velocity distribution of the particles becomes bimodal (fast particles in the kinks and slow particles resting in their respective potential minima). The threshold of $70 \%$ of the maximum velocity is chosen to lie in between these two peaks. The results differ only slightly on small variations of the threshold values.

\section{References}

1. Scholz, C. H. Earthquakes and friction laws. Nature 391, 37-42 (1998).

2. Persson, B. N. J. Theory of friction and boundary lubrication. Phys. Rev. B 48, 18140-18158 (1993).

3. Raviv, U. et al. Lubrication by charged polymers. Nature 425, 163-165 (2003).

4. Rubinstein, S. M., Cohen, G. \& Fineberg, J. Detachment fronts and the onset of dynamic friction. Nature 430, 1005-1009 (2004).

5. Frenkel, J. \& Kontorova, T. On the theory of plastic deformation and twinning. Physik. Z. Sowietunion 13, 1-10 (1938).

6. Kontorova, T. \& Frenkel, J. On the theory of plastic deformation and twinning. Zh. Eksp. Teor. Fiz. 8, 89-95 (1938).

7. Braun, O. M. \& Kivshar, Y. S. Nonlinear dynamics of the Frenkel-Kontorova model. Phys. Rep. 306, 1-108 (1998).

8. Braun, O. M. \& Kivshar, Y. S. The Frenkel-Kontorova Model: Concepts, Methods, and Applications (Springer, 2004).

9. Braun, O. M., Dauxois, T., Paliy, M. V. \& Peyrard, M. Mobility and diffusivity in a generalized Frenkel-Kontorova model. Phys. Rev. B 54, 321-331 (1996).

10. Braun, O. M., Bishop, A. R. \& Roder, J. Hysteresis in the underdamped driven Frenkel-Kontorova model. Phys. Rev. Lett. 79, 3692-3695 (1997).

11. Braun, O. M., Dauxois, T., Paliy, M. V. \& Peyrard, M. Nonlinear mobility of the generalized Frenkel-Kontorova model. Phys. Rev. E 55, 3598-3612 (1997).

12. Tekic, J., Braun, O. M. \& Hu, B. B. Dynamic phases in the two-dimensional underdamped driven Frenkel-Kontorova model. Phys. Rev. E 71, 026104 (2005).

13. Bowden, F. P. \& Tabor, D. Friction and Lubrication of Solids (Clarendon Press, 1950).
14. Lee, C. et al. Frictional characteristics of atomically thin sheets. Science 328, 76-80 (2010).

15. Dienwiebel, M. et al. Superlubricity of graphite. Phys. Rev. Lett. 92, 126101 (2004)

16. Hirano, M., Shinjo, K., Kaneko, R. \& Murata, Y. Observation of superlubricity by scanning tunneling microscopy. Phys. Rev. Lett. 78, 1448-1451 (1997).

17. Verhoeven, G. S., Dienwiebel, M. \& Frenken, J. W. M. Model calculations of superlubricity of graphite. Phys. Rev. B 70, 165418 (2004).

18. Filippov, A. E., Dienwiebel, M., Frenken, J. W. M., Klafter, J. \& Urbakh, M. Torque and twist against superlubricity. Phys. Rev. Lett. 100, 046102 (2008).

19. Janssen, T. Phonons and internal friction in incommensurate composites. J. Phys. Condens. Matter 14, 12411-12422 (2002).

20. Janssen, T., Radulescu, O. \& Rubtsov, A.N. Phasons, sliding modes and friction. Eur. Phys. J. B 29, 85-95 (2002).

21. Benassi, A., Vanossi, A. \& Tosatti, E. Nanofriction in cold ion traps. Nature Commun. 2, 236 (2011)

22. Mikhael, J., Roth, J., Helden, L. \& Bechinger, C. Archimedean-like tiling on decagonal quasicrystalline surfaces. Nature 454, 501-504 (2008).

23. Vanossi, A., Bishop, A. R. \& Bortolani, V. Role of substrate geometry in sliding friction. Nanotechnology 15, 790-794 (2004).

24. Park, J. Y. et al. High frictional anisotropy of periodic and aperiodic directions on a quasicrystal surface. Science 309, 1354-1356 (2005).

25. Dubois, J. M., Kang, S. S. \& Vonstebut, J. Quasi-crystalline low-friction coatings. J. Mater. Sci. Lett. 10, 537-541 (1991).

26. Filippov, A. E., Vanossi, A. \& Urbakh, M. Origin of friction anisotropy on a quasicrystal surface. Phys. Rev. Lett. 104, 074302 (2010).

27. Grimm, B., Hovel, H., Pollmann, M. \& Reihl, B. Physisorbed rare-gas monolayers: Evidence for domain-wall tilting. Phys. Rev. Lett. 83, 991-994 (1999).

28. Bleil, S., von Grunberg, H. H., Dobnikar, J., Castaneda-Priego, R. \& Bechinger, C. Strain-induced domain formation in two-dimensional colloidal systems. Europhys. Lett. 73, 450-456 (2006).

29. Vanossi, A. \& Braun, O. M. Driven dynamics of simplified tribological models. J. Phys. Condens. Matter 19, 35017 (2007).

30. Braun, O. M., Paliy, M. V., Roder, J. \& Bishop, A. R. Locked-to-running transition in the two-dimensional underdamped driven Frenkel-Kontorova model. Phys. Rev. E 63, 036129 (2001).

31. Vanossi, A., Roder, J., Bishop, A. R. \& Bortolani, V. Driven, underdamped Frenkel-Kontorova model on a quasiperiodic substrate. Phys. Rev. E 63, 017203 (2001).

32. Peyrard, M. \& Aubry, S. Critical-behaviour at the transition by breaking of analytiticity in the discrete Frenkel-Kontorova model. J. Phys. C 16, 1593-1608 (1983)

33. Brunner, M., Bechinger, C., Strepp, W., Lobaskin, V. \& von Grunberg, H. H. Density-dependent pair interactions in 2D colloidal suspensions. Europhys. Lett. 58, 926-932 (2002).

\section{Acknowledgements}

We thank V. Blickle for helpful discussions. This work is financially supported by the Deutsche Forschungsgemeinschaft (BE 1788-10).

\section{Author contributions}

T.B. carried out the experiment and analysed data, J.M. designed the experiment and C.B. designed the experiment and wrote the paper. 